\title{
Puberdade precoce central: perfil clínico e laboratorial de casos esporádicos e familiares em um centro de referência
}

\section{Central precocious puberty: clinical and laboratory profile of sporadic and familial cases in a referral center}

Mayara Ponte Madeira ${ }^{1,2}$. Iarley Ítalo Alves de Sousa ${ }^{1}$. Marina Mara de Sousa de Oliveira ${ }^{1}$. Isabelly de Oliveira Pinheiro ${ }^{1,2}$. Leylane Bernardes Forte ${ }^{1,2}$. Danielle de Souza Bessa ${ }^{1,2}$. Rosana Quezado ${ }^{1,2}$. Eveline Gadelha Pereira Fontenele ${ }^{1,2}$.

1 Universidade Federal do Ceará (UFC), Fortaleza, Ceará, Brasil. 2 Hospital Universitário Walter Cantídio (HUWC/UFC/ EBSERH), Fortaleza, Ceará, Brasil.

\section{RESUMO}

Objetivos: Analisar as características clínicas e laboratoriais de pacientes com puberdade precoce central (PPC) esporádica e familiar seguidos em um serviço de referência no nordeste do Brasil. Metodologia: Estudo descritivo, com análise retrospectiva dos prontuários, e estudo transversal, com análise de um questionário sobre consanguinidade e história familiar de puberdade precoce. Resultados: Sessenta e duas meninas com PPC foram incluídas. A manifestação inicial de puberdade mais comum foi telarca (73,8\%), que se encontrava no estágio M4 de Tanner em 46,2\%. A primeira consulta ocorreu aos 7,8 $\pm 1,0$ anos, em média 1,3 ano após o surgimento da telarca. A idade óssea era avançada em 2,2 $\pm 1,1$ anos. História familiar de PPC foi referida em $32,3 \%$ dos casos. O grupo de pacientes com PPC familiar apresentou menor idade de menarca materna, maior frequência de parto a termo, maior z-escore da altura inicial e maior hormônio luteinizante (LH) após a $1^{\text {a }}$ dose do análogo do GnRH que o grupo com PPC esporádica. Conclusões: O presente estudo identificou história familiar de PPC em 1/3 dos casos estudados. Essas pacientes apresentavam um perfil clínico e laboratorial distinto destacando-se a maior altura inicial e maiores valores de LH após estímulo. Estudos genéticos e epigenéticos futuros poderão contribuir para maior compreensão da etiopatogenia da PPC.

Palavras-chave: Puberdade precoce. Hormônio Luteizinante. Hormônio liberador de gonadotropina.

\section{ABSTRACT}

Objectives: To analyze the clinical and laboratory characteristics of patients with sporadic and familial central precocious puberty (CPP) followed at a reference service in northeastern Brazil. Methodology: Descriptive study, with retrospective analysis of medical records, and cross-sectional study, with analysis of a questionnaire on consanguinity and family history of precocious puberty. Results: Sixty-two girls with PPC were included. The most common initial manifestation of puberty was thelarche (73.8\%), which was in Tanner's M4 stage in 46.2\%. The first consultation took place at $7.8 \pm 1.0$ years, on average 1.3 year after the onset of the thelarche. Bone age was advanced by $2.2 \pm 1.1$ years. A family history of PCP was reported in $32.3 \%$ of cases. The group of patients with familial PCP had a lower age at maternal menarche, higher frequency of term delivery, higher initial height $\mathrm{z}$-score and higher luteinizing hormone (LH) after the 1st dose of the GnRH analogue than the group with sporadic PCP. Conclusions: The present study identified a family history of PCP in $1 / 3$ of the cases studied. These patients had a distinct clinical and laboratory profile, with a higher initial height and higher LH values after stimulation. Future genetic and epigenetic studies may contribute to a better understanding of the etiopathogenesis of PPC.

Keywords: Puberty, precocious. Luteiziniting hormone. Gonadotropin-releasing hormone.

Autor correspondente: Mayara Ponte Madeira, Universidade Federal do Ceará, Bloco dos ambulatórios (ilhas), Rua Coronel Nunes de Melo, s/n, Rodolfo Teófilo, Fortaleza, Ceará. CEP: 60430-270. Telefone: +55 85 3366-8165. E-mail: mayara.madeira@hotmail.com

Conflito de interesses: Não há qualquer conflito de interesses por parte de qualquer um dos autores.

Recebido em: 09 Set 2020; Revisado em: 17 Jun 2021; Aceito em: 21 Set 2021. 


\section{INTRODUÇÃO}

A puberdade é um processo fisiológico que envolve o aumento progressivo de hormônios sexuais, o desenvolvimento de caracteres sexuais secundários, a aceleração da velocidade de crescimento (VC) e culmina na aquisição de capacidade reprodutiva da vida adulta. Segundo os critérios descritos por Tanner para estadiar o desenvolvimento puberal, o início da puberdade (Tanner 2) é marcado pelo desenvolvimento mamário em meninas e aumento testicular em meninos (volume $\geq 4 \mathrm{ml}$ ). ${ }^{1}$ Esse processo é considerado precoce quando se inicia antes dos 8 anos em meninas e antes dos 9 anos em meninos. ${ }^{1}$

A puberdade precoce central (PPC) é dependente de gonadotrofinas e resulta da ativação prematura do eixo hipotálamo-hipofisário-gonadal, e os caracteres sexuais secundários são concordantes com o sexo do paciente (isossexual). ${ }^{2,3}$

Fatores genéticos estão envolvidos na determinação do início da puberdade e mutações em quatro genes (KISS1, KISS1R, MKRN3 e $D L K 1$ ) foram descritas na PPC. ${ }^{4,5}$ Por outro lado, algumas afecções neurológicas como hamartomas hipotalâmicos, tumores, malformações, inflamação e trauma do sistema nervoso central (SNC) podem determinar a precocidade sexual. ${ }^{3,6}$ Em meninos, particularmente, as causas neurológicas representam 2/3 dos casos de puberdade precoce (aproximadamente 50\% por tumores do SNC). ${ }^{3,6}$ Todavia, a forma idiopática é a mais comum, especialmente no sexo feminino (10:1).

A PPC é uma condição rara, com incidência variável de 1:5.000 - 1:10.000.3,6,7,8 No entanto, um estudo coreano demonstrou um aumento da incidência anual de PPC em meninas de 3,3 para 50,4 por 100.000 meninas; enquanto nos meninos, aumentou de 0,3 para 1,2 por 100.000 meninos. ${ }^{9}$ Estudos recentes demonstraram uma diminuição progressiva da idade de início da puberdade em crianças em todo o mundo. ${ }^{10-12}$ Embora a razão exata para essa mudança não seja completamente compreendida, geralmente é aceita como sendo o resultado de uma interação complexa entre fatores genéticos, endócrinos e ambientais. Sabe-se que a aceleração da industrialização em todo o mundo determinou um aumento significativo de contaminantes ambientais e a exposição a compostos químicos com ação estrogênica ou antiandrogênica (disruptores endócrinos) tem sido associada a mudanças no tempo da puberdade. ${ }^{12,13}$

O diagnóstico de PPC é confirmado pela dosagem de hormônio luteinizante $(\mathrm{LH})$ basal $>0,6 \mathrm{U} / \mathrm{L}$ (método imunofluorométrico, IFMA) ou $\geq 0,3 \mathrm{U} / \mathrm{L}$ (método quimioluminométrico, ICMA) em ambos os sexos. ${ }^{13}$ Assim como valores de LH $>8 \mathrm{UI} / \mathrm{L}$ (método IFMA ou ICMA) dosados 2 horas após a aplicação de 3,75 mg de um agonista do GnRH de longa duração (aGnRH) também confirmam o diagnóstico em ambos os sexos. ${ }^{6,14,15}$ A radiografia de mão e punho não dominante revela usualmente um avanço na idade óssea (IO) (método de Greulich \& Pyle $^{16}$ ), e habitualmente é utilizada para predizer a altura adulta pelo método de Bayley-Pinneau. ${ }^{17} \mathrm{~A}$ ultrassonografia pélvica em meninas auxilia na avaliação dos volumes uterino e ovarianos..$^{2,15}$ A ressonância magnética do
SNC, com ênfase na região hipotálamo-hipofisária, deve ser solicitada para detecção de tumores, malformações e outras afecções do SNC. ${ }^{15} \mathrm{O}$ tratamento da PPC consiste na aplicação intramuscular ou subcutânea de um aGnRH. O tratamento leva à dessensibilização e downregulation da sinalização do GnRH, reduzindo a liberação de gonadotrofinas (LH e hormônio folículo-estimulante (FSH)) e consequentemente dos esteroides sexuais.

O Serviço de Endocrinologia e Diabetes do Hospital Universitário Walter Cantídio (SED-HUWC) da Universidade Federal do Ceará é referência no atendimento aos portadores de puberdade precoce e desde 1997 disponibiliza o tratamento com os aGnRH em conformidade com o protocolo clínico e diretriz terapêutica do Ministério da Saúde (portaria $n^{0} 3 /$ SAS/MS de 8 de junho de 2017). Considerando que fatores genéticos estão envolvidos na patogênese da PPC, e que ainda não existem dados na literatura sobre a prevalência de PPC familiar em nosso meio, o objetivo do estudo é analisar o perfil demográfico e as características clínicas e laboratoriais de formas familiares e esporádicas entre portadores de PPC idiopática em um serviço de referência do Ceará.

\section{MATERIAIS E MÉTODOS}

Trata-se de um estudo de desenho misto, descritivo e transversal. $\mathrm{O}$ estudo descritivo consistiu na análise retrospectiva dos dados de prontuários de uma série de pacientes diagnosticados com PPC. O estudo transversal envolveu a análise dos dados de um questionário sobre consanguinidade e história familiar de puberdade precoce.

No estudo descritivo, foram incluídas todas as pacientes do sexo feminino com diagnóstico clínico e laboratorial de PPC e tratadas com aGnRH entre 1997 a 2019. No estudo transversal, foram incluídas somente as pacientes atualmente em seguimento e que não apresentavam uma causa orgânica para PPC (idiopática).

Foram excluídas todas pacientes adotivas ou que faziam seguimento irregular (intervalo entre consultas superior a seis meses), ou cujos prontuários não forneceram todos os dados clínicos e laboratoriais requeridos para análise.

A coleta de dados foi feita através do protocolo eletrônico elaborado na plataforma REDCap (REDCap 8.7.1 - (C) 2018 Vanderbilt University). As variáveis clínicas obtidas na primeira consulta foram: os sintomas iniciais referidos pelos pacientes e seus responsáveis na primeira consulta (sintomas auto-referidos), idade cronológica de aparecimento dos sinais puberais (em anos), estadiamento puberal pelo critério de Tanner (1 a 5) e os dados auxológicos: peso $(\mathrm{kg})$, estatura $(\mathrm{cm})$, índice de massa corpórea $\left(\mathrm{IMC}, \mathrm{kg} / \mathrm{m}^{2}\right.$ ). A altura foi comparada ao padrão populacional para idade e sexo do World Health Organization, 2021 para cálculo do escore Z. Para o IMC, em crianças entre 5 e 10 anos, foi utilizado o padrão da OMS (2007). ${ }^{18}$

Foram então categorizadas quanto à altura, como muito baixa estatura quando escore $\mathrm{Z}$ da altura $<-3$, baixa estatura quando 
escore $Z$ da altura $\geq-3$ e $<-2$ e estatura adequada quando escore $Z$ da altura encontra-se $\geq-2$. Em relação ao IMC para idade, foram classificadas conforme o escore $\mathrm{Z}$ do IMC em magreza acentuada $<-3$, magreza $\geq-3$ e $<-2$, eutrofia $\geq-2$ e $\leq+1$, sobrepeso $\geq+1$ e $\leq+2$, obesidade $\geq+2$ e $\leq+3$ e obesidade grave $\geq+3$.

Quanto aos exames obtidos a partir dos registros dos prontuários, foram considerados para análise somente os valores de LH basal e/ou após estímulo com GnRH/aGnRH obtidos pelo mesmo método (ICMA, em UI/L), e os laudos de idade óssea, ultrassonografia pélvica e ressonância nuclear magnética do crânio. A idade óssea estimada pelo método de Greulich \& Pyle ${ }^{17}$ foi considerada avançada quando estava 1 ano acima da idade cronológica. As medidas de volume ovariano $(>1,5 \mathrm{~mL})$ e comprimento uterino $(>3,4 \mathrm{~cm})$ foram consideradas como púberes. ${ }^{15}$

Um questionário sobre consanguinidade, história familiar (HF) de puberdade precoce e idade da menarca da mãe foi aplicado entre as portadoras de PPC idiopática em seguimento atual. A PPC familiar foi definida pela presença de dois ou mais membros afetados na família. As pacientes que não relataram história familiar de puberdade precoce foram classificadas como casos esporádicos.

Este estudo foi conduzido no Ambulatório de Gônadas e Desenvolvimento, no Hospital Universitário Walter Cantídio, de acordo com as boas práticas clínicas definidas pelo International Conference on Harmonization (ICH) e com a resolução número 466 de 12 de dezembro 2012 do Conselho Nacional de Saúde. O protocolo clínico foi aprovado pelo Comitê de Ética e Pesquisa do hospital (número do parecer 3.311.607) e todos pacientes e seus pais ou responsáveis legais incluídos no estudo deram seu assentimento ou consentimento livre e esclarecido por escrito.

\section{ANÁLISE ESTATÍSTICA}

Os dados foram processados na plataforma REDCap e exportados para programa de estatística Statistical Package for the Social Sciences (SPSS), versão 22.0 (USA) e software $\mathrm{R}$ 3.3.1. As variáveis contínuas foram expressas em média e desvio-padrão ou mediana e quartis conforme o tipo de distribuição. As variáveis categóricas foram apresentadas como frequência (\%). A análise comparativa dos grupos foi feita pelo teste t de Student, teste U de Mann-Whitney ou teste de Kruskall-Wallis para as variáveis contínuas, condicionado à aderência dos dados à distribuição gaussiana. E a associação entre as variáveis categóricas foi realizada pelo teste de qui-quadrado de Pearson e teste exato de Fisher. O nível de significância utilizado foi de 5\%.

\section{RESULTADOS}

Foram convidados a participar do estudo 84 pacientes com diagnóstico de PPC. Foram excluídos dois pacientes do sexo masculino. Também foram excluídas 18 do sexo feminino, por não responderem o questionário, e duas pacientes, por serem adotadas. Ao final, 62 pacientes do sexo feminino foram incluídas no estudo. A idade média na primeira consulta foi 7,81 $\pm 1,05$ anos, e o tempo decorrido desde a primeira manifestação clínica de puberdade e a primeira consulta no serviço foi de 1,47 $\pm 1,08$ anos. A maioria era procedente de Fortaleza (49,3\%) ou Caucaia $(10,4 \%)$, possuía renda familiar acima de 1 saláriomínimo $(64,6 \%)$, nascida a termo $(66,1 \%)$ e de parto cesariano (74,2\%). Cinco pacientes $(7,7 \%)$ possuíam pais consanguíneos.

A manifestação inicial de puberdade mais comumente relatada foi telarca $(73,8 \%)$ que se encontrava no estágio M4 de Tanner em 46,2\%. A telarca surgiu com idade média de 6,5 $\pm 1,24$ anos. A pubarca foi a manifestação inicial em 12,3\% com idade média de $6,94 \pm 1,43$ anos. Nenhuma paciente apresentou menarca como manifestação inicial. O escore $\mathrm{Z}$ da altura e de IMC aferidos foram $0,93 \pm 0,94$ e $0,88 \pm$ 1,04 , respectivamente. Os sintomas iniciais auto-referidos mais comuns foram alteração de comportamento $(24,2 \%)$ e alteração visual (19,4\%) (Gráfico 1).

O valor médio de LH basal foi 1,68 $\pm 2,04 \mathrm{UI} / \mathrm{L}$, sendo $\geq 0,3$ $\mathrm{UI} / \mathrm{L}$ em $80 \%$ das pacientes e após $1^{\text {a }}$ dose de aGnRH $>8 \mathrm{UI} / \mathrm{L}$ em 63,6\% (ICMA). A idade óssea estava avançada em 2,2 \pm 1,15 anos no diagnóstico. O volume uterino, ovariano direito e esquerdo médio medidos foram de 7,28 $\pm 5 \mathrm{~cm}^{3}, 3,15 \pm 1,88$ $\mathrm{cm}^{3}$ e 2,97 $\pm 1,67 \mathrm{~cm}^{3}$, respectivamente. A história familiar de PPC foi referida em $32,3 \%$ das pacientes $(n=20)$, sendo a maioria (55\%) de origem materna (Gráfico 2).

Gráfico 1. Frequência de sintomas referidos na consulta inicial por pacientes com puberdade precoce central $(n=62)$.

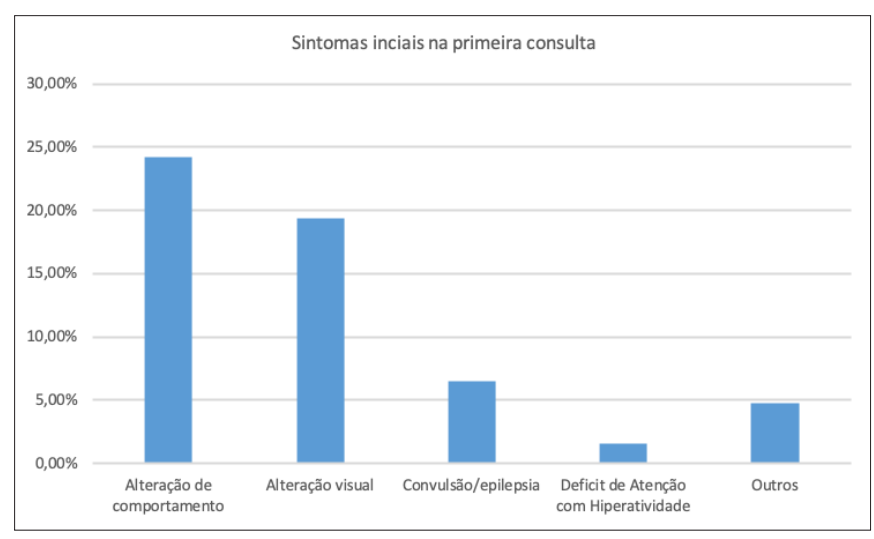

Gráfico 2. Frequência de história familiar de puberdade precoce central $(n=62)$.

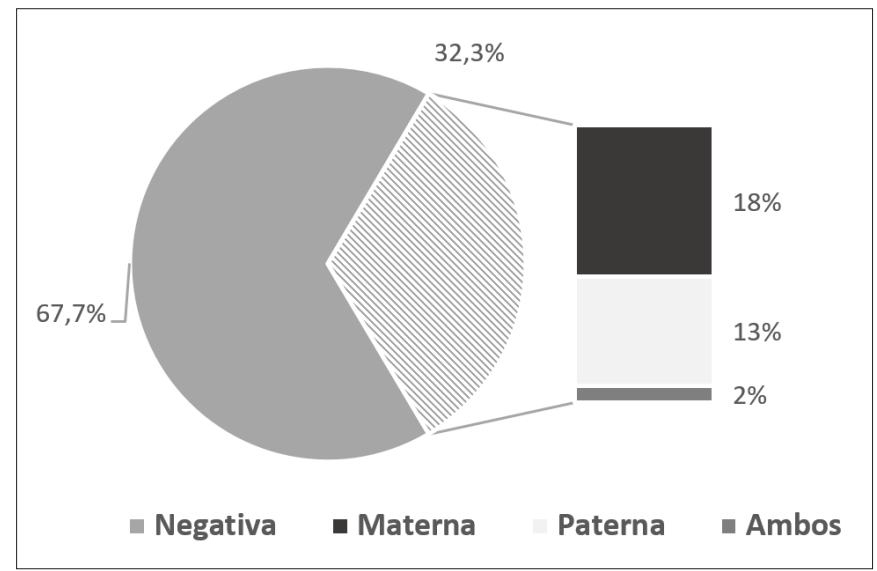


Analisando os dados clínicos das pacientes com HF de PPC, foi observado que a média do escore $\mathrm{Z}$ de altura na primeira consulta foi significativamente maior nas pacientes com HF positiva (1,22 $\pm 0,71$ vs $0,72 \pm 0,98, p=0,029)$ (Tabela 1). Este grupo também apresentou menor idade da menarca materna $(11,4 \pm 1,5 v s 12,4 \pm$ $1,5, p=0,05)$ e menor frequência de parto pré-termo e pós-termo que no grupo com PPC esporádica com significância estatística $(p=0,008)$ (Tabela 1). Não foram detectadas diferenças estatisticamente significantes entre os dois grupos quanto à escolaridade, renda familiar, idade cronológica na $1^{\text {a }}$ consulta, idade de telarca ou pubarca, estádio puberal na consulta inicial ou tempo decorrido entre o primeiro sinal puberal e a $1^{\text {a }}$ consulta.

Quanto aos exames complementares, o valor médio de pico de LH após $1^{\mathrm{a}}$ dose do aGnRH foi significativamente maior no grupo das pacientes com HF positiva $(29,1 \pm 24,4$ vs $17,8 \pm 18,8, p=0,048$ ) (Figura 1). Não houve diferença estatisticamente significativa entre os grupos quanto aos valores basais de LH, avanço de idade óssea, volume uterino e dos ovários no momento da primeira consulta.
Figura 1. Valor de pico de LH após $1^{\text {a }}$ dose de análogo de GnRH nas pacientes de acordo com a presença de história familiar de PPC.

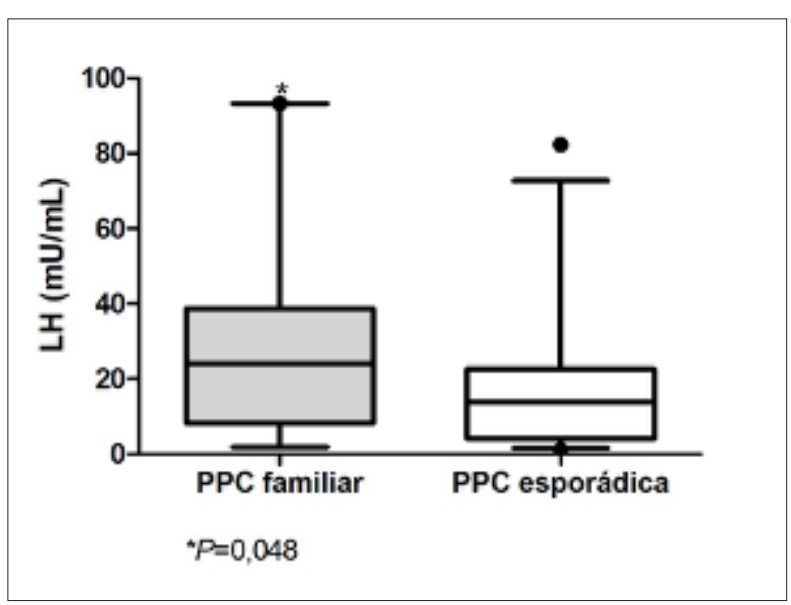

$\mathrm{LH}=$ hormônio luteinizante; $\mathrm{PPC}=$ puberdade precoce central.

Tabela 1. Dados clínicos e laboratoriais das pacientes de acordo com a história familiar de PPC (n=62).

\begin{tabular}{|c|c|c|}
\hline Variável & HF de PPC ausente & HF de PPC presente \\
\hline Pacientes, n (\%) & $42(67,7 \%)$ & $20(32,3 \%)$ \\
\hline Idade na $1^{\mathrm{a}}$ consulta (a) & $7,93 \pm 1,04$ & $7,6 \pm 1,1$ \\
\hline Idade de pubarca (a) & $7,10 \pm 1,61$ & $6,7 \pm 1,2$ \\
\hline Idade de telarca (a) & $6,60 \pm 1,15$ & $6,2 \pm 1,4$ \\
\hline $\mathrm{Z}$ altura & $0,72 \pm 0,98$ & $1,22 \pm 0,71^{*}$ \\
\hline Z IMC & $0,75 \pm 1,14$ & $1,02 \pm 0,74$ \\
\hline Peso ao nascer (g) & $3,067 \pm 647$ & $3,131 \pm 602$ \\
\hline Idade menarca (mãe) (a) & $12,4 \pm 1,5$ & $11,4 \pm 1,49^{*}$ \\
\hline $\mathrm{Z}$ altura-alvo & $-1,01 \pm 0,75$ & $-1,01 \pm 0,7$ \\
\hline Idade óssea (a) & $9,71 \pm 1,67$ & $9,8 \pm 1,53$ \\
\hline Avanço de idade óssea (a) & $2,0 \pm 1,09$ & $2,4 \pm 1,14$ \\
\hline Volume uterino $\left(\mathrm{cm}^{3}\right)$ & $6,9 \pm 4,8$ & $6,9 \pm 4,1$ \\
\hline Volume ovário D $\left(\mathrm{cm}^{3}\right)$ & $3,0 \pm 1,8$ & $3,1 \pm 1,8$ \\
\hline Volume ovário E $\left(\mathrm{cm}^{3}\right)$ & $2,9 \pm 1,8$ & $2,8 \pm 1,2$ \\
\hline LH basal (mUI/mL) & $1,76 \pm 2,1$ & $1,65 \pm 2,05$ \\
\hline LH pós $1^{\mathrm{a}}$ dose $(\mathrm{mUI} / \mathrm{mL})$ & $17,8 \pm 18,8$ & $29,1 \pm 24,4^{*}$ \\
\hline LH pós $6^{\mathrm{a}}$ dose $(\mathrm{mUI} / \mathrm{mL})$ & $3,1 \pm 2,7$ & $3,25 \pm 2,67$ \\
\hline Pré-termo / Pós-termo & $18(42,9 \%)$ & $2(10 \%)$ \\
\hline A termo & $24(57,1 \%)$ & $18(90 \%)$ \\
\hline
\end{tabular}

$* \mathrm{p}<0,05$

$\mathrm{a}=$ anos; $\mathrm{D}=$ direito; $\mathrm{E}=$ esquerdo; $\mathrm{g}=$ gramas; $\mathrm{HF}=$ história familiar; $\mathrm{IMC}=$ índice de massa corpórea; $\mathrm{LH}=$ hormônio luteinizante; $\mathrm{PPC}=$ puberdade precoce central; $\mathrm{Z}=$ escore padrão. 


\section{DISCUSSÃO}

O presente estudo avaliou o perfil clínico-laboratorial de pacientes do sexo feminino com diagnóstico de PPC em um serviço de referência do nordeste brasileiro.

A idade média de surgimento do primeiro sinal puberal foi 6,5 anos, semelhante aos estudos de Aguiar, ${ }^{19}$ Chemaitillys $^{20} \mathrm{e}$ Adan, ${ }^{21}$ cujas idades foram 6,3, 6,6 e 6,4 anos, respectivamente. No entanto, o tempo decorrido entre o aparecimento dos sinais iniciais e a consulta inicial foi em média de 1,47 ano e o avanço da idade óssea no início do tratamento foi em média de 2,2 anos, superior ao encontrado por Chemaitilly, ${ }^{20}$ que observou avanço de idade óssea de apenas 1,4 ano. Por outro lado, nossos achados se assemelham ao observado por Aguiar e colaboradores ${ }^{19}$ em um estudo realizado no Nordeste do Brasil, que observou um tempo entre a percepção do início da puberdade e a primeira consulta de um 1,25 ano, em média, e um avanço médio de idade óssea de 2,4 anos. Esse achado ressalta a importância de se aprimorar o referenciamento das unidades de atenção básica ao serviço especializado para assegurar que o diagnóstico e o tratamento possam ser instituídos em tempo hábil na rede pública de saúde.

Os sintomas iniciais auto-referidos mais comuns foram alteração de comportamento $(24,2 \%)$ e alteração visual $(19,4 \%)$. As alterações de comportamento eram inespecíficas e descritas como agitação, mudança de humor, alteração no padrão de sono. A alteração visual mais relatada foi alterações de refração. Esses sintomas, embora frequentes, não estavam acompanhados de outras manifestações clínicas ou sinais neurológicos que justificassem a necessidade de maior investigação.

No presente estudo, foi observado um valor médio de $\mathrm{LH}$ basal de 1,68 UI/L e após estímulo com aGnRH de 21,1 UI/L (ICMA). Embora os valores sejam comparáveis aos publicados na literatura, existe uma grande variabilidade entre os estudos, o que pode ser atribuído à diferença entre os ensaios utilizados e a população estudada (Tabela 2).

Tabela 2. Variabilidade dos valores médios de LH basal e após estímulo com análogo de GnRH entre diferentes estudos

\begin{tabular}{llll}
\hline Autor, ano & LH basal (UI/L) & LH pós aGnRH (UI/L) & Método \\
\hline Brito et al, 2004 & 0,6 & 10,0 & IFMA \\
Aguiar et al, 2005 & 2,2 & 19 & RIE \\
Park e Kim, 2017 & 0,74 & 12,1 & ICMA \\
Fujita et al, 2019 & 4,8 & 27,1 & ICMA \\
Madeira et al, 2020 & 1,68 & 21,1 & ICMA \\
\hline
\end{tabular}

aGnRH = análogo de GnRH; ICMA = quimioluminescência; IFMA = imunofluorimétrico; LH = hormônio luteinizante; $\mathrm{RIE}=$ radioimunoensaio.

Em nosso estudo evidenciamos uma prevalência de 32,3\% de relato de história familiar de PPC, semelhante ao observado por De Vries ${ }^{22}$ que identificou uma prevalência de $27,5 \%$ de formas familiares. Em nossa amostra, identificamos também uma menor idade da menarca materna no grupo de pacientes com HF. Essa correspondência entre idade de menarca entre mães e filhas, a maior correlação na idade na menarca entre gêmeas monozigóticas do que gêmeas dizigóticas ou pares de irmãs e entre membros de um mesmo grupo étnico tem sido evidenciada em diversos estudos. Estudos de associação ampla do genoma demonstram que a contribuição dos fatores genéticos explica 60 a $80 \%$ da variabilidade observada no tempo da puberdade. ${ }^{23-25}$

A maioria das pacientes apresentou valores de LH após $1^{\text {a }}$ dose do aGnRH $>8 \mathrm{UI} / \mathrm{L}$. No entanto, nas pacientes com HF de PPC esses valores foram significativamente superiores ao observado no grupo de pacientes sem HF $(29,1 \pm 24,4$ vs 17,8 $\pm 18,8, p=0,048)$, com $78,9 \%$ das pacientes $>8 \mathrm{UI} / \mathrm{L}$, embora o valor de LH basal não tenha havido diferença significativa. Esses maiores níveis de LH pós-aGnRH podem sugerir maior número e amplitude dos pulsos de secreção das gonadotrofinas nesses pacientes.
Os pacientes com relato de HF de PPC apresentaram Z-escore de altura mais elevado $(1,22 \pm 0,71$ vs $0,72 \pm 0,98$, $p=0,029)$ na apresentação inicial, sem diferença entre os grupos quanto ao $\mathrm{Z}$ da altura-alvo. A estatura na puberdade precoce geralmente está acima do padrão familiar. $\mathrm{Na}$ PPC, o avanço da IO é desproporcionalmente maior que o aumento da VC, promovendo fechamento prematuro da cartilagem de crescimento, o que pode determinar perda da estatura final. ${ }^{26}$ Houve maior frequência de parto prétermo e pós-termo nas pacientes com PPC esporádica ( $p=$ 0,008 ) (Tabela 1), no entanto, não há consenso na literatura quanto à influência da idade gestacional sobre o tempo de puberdade. ${ }^{27}$

O presente estudo demonstrou uma elevada frequência de formas familiares, corroborando a importância da análise genética dos casos familiares de PPC. Esse estudo, no entanto, apresenta algumas limitações. Primeiramente, o tamanho e característica da amostra (sexo feminino e pacientes atendidas em um serviço de referência) não permite extrapolar os resultados para a população geral. A coleta dos dados de idade de menarca materna e história familiar de PPC ficaram sujeitos aos possíveis vieses de memória 
do entrevistado. Os dados analisados dos prontuários de exames realizados, como ultrassonografia pélvica e radiografia para idade óssea, foram realizados em diferentes locais e por diferentes examinadores, podendo ter vieses na uniformização das informações. Além disso, por se tratar de uma análise transversal e retrospectiva, não foram analisados prospectivamente os desfechos nos dois grupos de PPC ao final do tratamento com aGnRH, não sendo possível inferir se as diferenças observadas na consulta inicial nas pacientes com HF irão se refletir em diferenças na estatura final e idade de menarca.

\section{REFERÊNCIAS}

1. Eugster E. Consensus Statement on the Use of GonadotropinReleasing Hormone Analogs in Children. Yearbook of Medicine. 2009;2009:555-6. http://dx.doi.org/10.1016/s00843873(09)79462-3

2. Saenger P, Snyder P, Kirkland J, Crowley W, Hoppin A, Martin K. Overview of precocious puberty [Internet]. UpToDate; 2009. Disponível em: https://www.uptodate.com/contents/definitionetiology-and-evaluation-of-precocious-puberty. Acesso em: $01 / 10 / 2019$.

3. Carel JC, Léger J. Clinical practice. Precocious puberty. N Engl J Med. 2008;358(22):2366-77.

4. Maione L, Bouvattier C, Kaiser UB. Central precocious puberty: Recent advances in understanding the aetiology and in the clinical approach. Clin Endocrinol (Oxf) . 2021 Oct;95(4):542-555.

5. Roberts SA, Kaiser UB. Genetics in Endocrinology: Genetic etiologies of central precocious puberty and the role of imprinted genes. Eur J Endocrinol. 2020;183(4):R107-17.

6. Macedo DB, Cukier P, Mendonca BB, Latronico AC, Brito VN. Avanços na etiologia, no diagnóstico e no tratamento da puberdade precoce central Arq Bras Endocrinol Metab.2014;58(2):108-17.

7. Abreu AP, Kaiser UB. Pubertal development and regulation. Lancet Diabetes Endocrinol. 2016;4(3):254-264.

8. Partsch C-J, Sippell WG. Pathogenesis and epidemiology of precocious puberty. Effects of exogenous oestrogens. Hum Reprod Update. 2001;7(3):292-302.

9. Kim SH, Huh K, Won S, Lee K-W, Park M-J. A Significant Increase in the Incidence of Central Precocious Puberty among Korean Girls from 2004 to 2010. PLoS One. 2015;10(11):e0141844.

10. Louis GM, Buck Louis GM, Earl Gray L, Marcus M, Ojeda SR, Pescovitz $\mathrm{OH}$, et al. Environmental Factors and Puberty Timing: Expert Panel Research Needs. Pediatrics. 2008;121(Suppl 3):S192207.

11. Nebesio TD, Pescovitz OH. The Role of Endocrine Disruptors in Pubertal Development. In: Pescovitz OH, Walvoord EC. (eds) When Puberty is Precocious. Contemporary Endocrinology. [s.1.]: Humana Press; 2007. p. 425-42.

\section{CONCLUSÃO}

$\mathrm{O}$ presente estudo descreveu as características clínicas e laboratoriais de meninas com PPC tratadas com aGnRH em um serviço de referência do Ceará. A presença de história familiar de PPC foi encontrada em $1 / 3$ da amostra. Essas pacientes apresentavam maior altura inicial e maiores valores de LH após estímulo. O grande avanço de idade óssea observado na consulta inicial em todas as pacientes ressalta a importância de um referenciamento mais precoce ao serviço especializado para reduzir o comprometimento da estatura final. Estudos genéticos e epigenéticos futuros poderão contribuir para maior compreensão da etiopatogenia da PPC em nosso meio.

12. Dickman EJ, Lee MM. The influence of endocrine disruptors on pubertal timing. Curr Opin Endocrinol Diabetes Obes. 2009;16(1):2530 .

13. Brito VN, Latronico AC, Arnhold IJP, Mendonça BB. Update on the etiology, diagnosis and therapeutic management of sexual precocity. Arq Bras Endocrinol Metab. 2008;52(1):18-31.

14. Latronico AC, Brito VN, Carel J-C. Causes, diagnosis, and treatment of central precocious puberty. Lancet Diabetes Endocrinol. 2016;4(3):265-74.

15. Brasil, Ministério da Saúde. Protocolo clínico e diretrizes terapêuticas: protocolo clínico e diretrizes terapêuticas puberdade precoce central. [internet]. Brasília (DF): Ministério da Saúde; 2017. Disponível em: https://portalarquivos2.saude.gov.br/images/ pdf/2017/julho/03/PCDT-Puberdade-Precoce-Central_08_06_2017. pdf. Acesso em: 17 set. 2018.

16. Dahlberg PS, Mosdøl A, Ding Y, Bleka Ø, Rolseth V, Straumann $\mathrm{GH}$, et al. A systematic review of the agreement between chronological age and skeletal age based on the Greulich and Pyle atlas. Eur Radiol. 2019;29(6):2936-48.

17. Bar A, Linder B, Sobel EH, Saenger P, DiMartino-Nardi J. BayleyPinneau method of height prediction in girls with central precocious puberty: correlation with adult height. J Pediatr. 1995;126(6):955-8.

18. World Health Organization. Epidemiological; Growth reference data for 5-19 years [Internet]. [s.1.], 2021. Disponível em: https:// www.who.int/toolkits/growth-reference-data-for-5to19-years. Acesso em: 17 set. 2021.

19. Aguiar AL, Couto-Silva AC, Freitas I, Cruz T, Rodrigues M, Adan LF. Arq Bras Endocrinol Metab. 2005;49(4):536-41.

20. Chemaitilly W, Trivin C, Adan L, Gall V, Sainte-Rose C, Brauner R. Central precocious puberty: clinical and laboratory features. Clin Endocrinol . 2001;54(3):289-94.

21. Adan L, Chemaitilly W, Trivin C, Brauner R. Factors predicting adult height in girls with idiopathic central precocious puberty: implications for treatment. Clin Endocrinol . 2002;56(3):297-302.

22. Vries L de, de Vries L, Kauschansky A, Shohat M, Phillip M. Familial Central Precocious Puberty Suggests Autosomal Dominant Inheritance. J Clin Endocrinol Metab. 2004;89(4):1794-800. 
23. Palmert MR, Boepple PA. Variation in the timing of puberty: clinical spectrum and genetic investigation. J Clin Endocrinol Metab. 2001;86(6):2364-8.

24. Parent A-S, Teilmann G, Juul A, Skakkebaek NE, Toppari J, Bourguignon J-P. The Timing of Normal Puberty and the Age Limits of Sexual Precocity: Variations around the World, Secular Trends, and Changes after Migration. Endocr Rev. 2003;24(5):668-93.

25. Brito VN, Latronico AC, Arnhold IJP, Mendonca BB. A single luteinizing hormone determination 2 hours after depot leuprolide is useful for therapy monitoring of gonadotropin-dependent precocious puberty in girls. J Clin Endocrinol Metab. 2004;89(9):4338-42.

26. Park J, Kim JH. Change in body mass index and insulin resistance after 1-year treatment with gonadotropin-releasing hormone agonists in girls with central precocious puberty. Ann Pediatr Endocrinol Metab. 2017;22(1):27-35.

27. James E, Wood CL, Nair H, Williams TC. Preterm birth and the timing of puberty: a systematic review. BMC Pediatr. 2018;18(1):3.

\section{Como citar:}

Madeira MP, Sousa II, Oliveira MM, Pinheiro IO, Forte LB, Bessa DS, et al. Puberdade precoce central: perfil clínico e laboratorial de casos esporádicos e familiares em um centro de referência. Rev Med UFC. 2021;61(1):1-7. 